\title{
A note on identities with derivations in prime rings
}

\author{
NADEEM UR REHMAN*
}

\begin{abstract}
The present paper's primary purpose is to study the connection between commutativity of rings and the behavior of its derivations in prime rings.
\end{abstract}

\section{INTRODUCTION}

Throughout this article, unless stated otherwise, $\mathfrak{R}$ will be associative ring. $Z(\mathfrak{R})$ the center of $\mathfrak{R}$, Martindale quotient ring $\mathcal{Q}, \mathcal{U}$ its Utumi quotient ring and the extended centroid $\mathfrak{C}$. For $\mathfrak{x}, \mathfrak{y} \in \mathfrak{R}$ and each $n \geq 0$, set $[\mathfrak{x}, \mathfrak{y}]_{0}=\mathfrak{x},[\mathfrak{x}, \mathfrak{y}]_{1}=\mathfrak{x} \mathfrak{y}-\mathfrak{y x}$, then an Engel condition is a polynomial $[\mathfrak{x}, \mathfrak{y}]_{m}=\left[[\mathfrak{x}, \mathfrak{y}]_{m-1}, \mathfrak{y}\right], m=1,2, \ldots$, in noncommuting indeterminate. The ring $\mathfrak{R}$ satisfies an Engel condition if there exists positive integer $m$ such that $[\mathfrak{x}, \mathfrak{y}]_{m}=0$. Recall that a ring $\mathfrak{R}$ is prime if for and $a, b \in \mathfrak{R}, a \mathfrak{R} b=(0)$ implies $a=0$ or $b=0$, and a semiprime if for any $a \in \mathfrak{R}, a \mathfrak{R} a=(0)$ implies $a=0$. An additive mapping $\mathfrak{F}: \mathfrak{R} \rightarrow \mathfrak{R}$ is called a derivation if $\mathfrak{F}(\mathfrak{x} \mathfrak{y})=\mathfrak{F}(\mathfrak{x}) \mathfrak{y}+\mathfrak{x} \mathfrak{F}(\mathfrak{y})$ holds for all $\mathfrak{x}, \mathfrak{y} \in \mathfrak{R}$. In particular, $\mathfrak{F}$ is an inner derivation induced by an element $a \in \mathfrak{R}$, if $\mathfrak{F}_{a}=[a, \mathfrak{x}]$ for all $\mathfrak{x} \in \mathfrak{R}$. Moreover, $\mathfrak{F}$ is said to be $\mathcal{Q}$-inner if the extension of $\mathfrak{F}$ to $\mathcal{Q}$ is inner, otherwise $\mathcal{Q}$-outer.

A classical result of ring theory established by Jacobson [6] generalizes at the same time the Theorem of Wedderburn that "every finite division ring is commutative" and also the result that any Boolean ring is commutative ring. The theorem to which we refer is, namely: "Any ring in which $\mathfrak{x}^{n}=\mathfrak{x}$, $n>1$ is a fixed integer, is necessarily commutative." Further, Herstein [4] established that "a ring must be commutative if it satisfied $[\mathfrak{x}, \mathfrak{y}]^{n}=[\mathfrak{x}, \mathfrak{y}]$ for every $\mathfrak{x}, \mathfrak{y} \in \mathfrak{R}, n>1$ is a fixed integer." In 2011 Huang [5] discussed the commutativity of prime ring and prove that "if a prime ring $\mathfrak{R}$ admits

2020 Mathematics Subject Classification. Primary: 16N60; Secondary: 16W25; 16 U80.

Key words and phrases. Ideal, Martindale ring of quotients, prime ring, generalized polynomial identity (GPI).

Full paper. Received 1 July 2019, revised 20 February 2020, revised 11 November 2020, accepted 28 November 2020, available online 16 March 2021.

* This research is supported by the National Board of Higher Mathematics (NBHM), India, Grant No. 02011/16/2020 NBHM (R. P.) R \& D II/ 7786. 
a derivation $\mathfrak{F}$ such that $\mathfrak{F}([\mathfrak{x}, \mathfrak{y}])^{m}=[\mathfrak{x}, \mathfrak{y}]_{n}$ for all $\mathfrak{x}, \mathfrak{y} \in \mathfrak{L}$ the nonzero ideal of $\mathfrak{R}$, where $m, n>1$ are integers, must be commutative." On the other hand Giambruno et al. [3] generalized Herstein result for Engle type condition and they established that "a ring must be commutative if it satisfies $\left([\mathfrak{x}, \mathfrak{y}]_{m}\right)^{n}=[\mathfrak{x}, \mathfrak{y}]_{m} . "$ Recently, the author with Raza [11] obtained that "a prime ring is commutative if it satisfies $\mathfrak{F}\left([\mathfrak{x}, \mathfrak{y}]_{m}\right)^{n}=[\mathfrak{x}, \mathfrak{y}]_{m}$ for all $\mathfrak{x}, \mathfrak{y} \in \mathfrak{L}$, a nonzero ideal of $\mathfrak{R}$." Motivated by these results, in the present article, we study prime and semiprime rings $\mathfrak{R}$ admitting a derivation $\mathfrak{F}$ satisfying the condition

$$
\left[\mathfrak{F}\left([\mathfrak{x}, \mathfrak{y}]_{m}\right),[\mathfrak{x}, \mathfrak{y}]_{m}\right]^{n}=\left[\mathfrak{F}\left([\mathfrak{x}, \mathfrak{y}]_{m}\right),[\mathfrak{x}, \mathfrak{y}]_{m}\right],
$$

for all $\mathfrak{x}, \mathfrak{y}$ in some appropriate subset of $\mathfrak{R}$.

\section{The Results}

In what follows, unless stated otherwise, $\mathfrak{R}$ will be a prime ring. We denote the Matrindale quotient ring of $\mathfrak{R}$ by $\mathcal{Q}$. Let $\mathfrak{C}$ be the center of $\mathcal{Q}$, which is called the extended centroid of $\mathfrak{R}$. The definitions, the axiomatic formulations and the properties of these quotient ring can be found [1]. Note that $\mathcal{Q}$ is also prime ring with identity and $\mathfrak{C}$ is a field.

Before proving our main theorem, we will fix some notations and collect some known result which we will use in the sequel.

Fact 2.1. "Let $\mathfrak{R}$ be a prime ring and $\mathfrak{L}$ a two sided ideal of $\mathfrak{R}$. Then $\mathfrak{L}, \mathfrak{R}$, $\mathcal{U}$ satisfy the same generalized polynomial identities with coefficients in $\mathcal{U}$ " (see [2]).

Fact 2.2. "Every derivation $\mathfrak{F}$ of $\mathfrak{R}$ can be uniquely extended to a derivation on $\mathcal{U}$ " (see Proposition 2,5,1 of [1]).

Fact 2.3. "Let $\mathfrak{R}$ be a prime ring and $\mathfrak{L}$ a two sided ideal of $\mathfrak{R}$. Then $\mathfrak{R}, \mathfrak{L}$ and $\mathcal{U}$ satisfy the same differential identities" (see [9]).

We are now ready to state the main result.

Theorem 1. Let $\mathfrak{R}$ be a prime ring of characteristic different from two, $\mathfrak{L}$ be a nonzero idela of $\mathfrak{R}$ and $\mathfrak{F}$ be a nonzero derivation of $\mathfrak{R}$. If $\mathfrak{L}$ satisfies

$$
\left[\mathfrak{F}\left([\mathfrak{x}, \mathfrak{y}]_{m}\right),[\mathfrak{x}, \mathfrak{y}]_{m}\right]^{n}=\left[\mathfrak{F}\left([\mathfrak{x}, \mathfrak{y}]_{m}\right),[\mathfrak{x}, \mathfrak{y}]_{m}\right],
$$

for all $\mathfrak{x}, \mathfrak{y} \in \mathfrak{L}$, then $\mathfrak{R}$ is commutative.

Proof. In the light of Kharchenko's theory [7], we split our proof into two cases. Firstly, we assume that $\mathfrak{F}$ is an $\mathcal{Q}$-inner derivation induced by an element $\lambda \in \mathcal{Q}$, i.e., $\mathfrak{F}(\mathfrak{x})=[\lambda, \mathfrak{x}]$ for all $\mathfrak{x} \in \mathfrak{R}$, then we have

$$
\left[\left[\lambda,[\mathfrak{x}, \mathfrak{y}]_{m}\right],[\mathfrak{x}, \mathfrak{y}]_{m}\right]^{n}=\left[\left[\lambda,[\mathfrak{x}, \mathfrak{y}]_{m}\right],[\mathfrak{x}, \mathfrak{y}]_{m}\right],
$$

for all $\mathfrak{x}, \mathfrak{y} \in \mathfrak{L}$. If $\lambda \in \mathfrak{C}$, then we are done. Thus we may assume that $\lambda \notin \mathfrak{C}$. In this case (1), by Chuang [2, Theorem 2], this identity is a non-trivial generalized polynomial identity for $\mathcal{Q}$ as $\lambda \notin \mathfrak{C}$. Thus, by Matrindate's 
[10], $\mathcal{Q}$ is a primitive ring which is isomorphic to a dense ring of linear transformations of a vector space $\mathfrak{V}$ over $\mathfrak{C}$. Suppose first that $\operatorname{dim}_{\mathfrak{C}}(\mathfrak{V})=l$, where $l \geq 3$ a finite positive integer. Then we may assume that $\mathfrak{M}_{l}(\mathfrak{C})$, the full matrix ring of all $l \times l$ matrices over $\mathfrak{C}$. As usual, by $e_{i j}, 1 \leq i, j \leq l$, we denote the matrix unit whose $(i, j)$-entry is equal to 1 and all its other entries are equal to zero. Let $\mu=\sum_{i j} \alpha_{i j} e_{i j}$. We show that $\mu$ is a diagonal matrix. Let $\mathfrak{x}=e_{i j}$ and $\mathfrak{y}=e_{j j}$. In this case we deduce that

$$
\left(\left[\left[\lambda, e_{i j}\right], e_{i j}\right]\right)^{n}=\left[\left[\lambda, e_{i j}\right], e_{i j}\right],
$$

that is, $e_{i j} \lambda e_{i j}=0$ and hence $\alpha_{j \mathrm{i}}=0$, for any $i \neq j$. Therefore $\mu$ is a diagonal matrix. In the next step we will prove that $\mu$ is central matrix. Since $\mu$ is a diagonal matrix. Moreover, if there exists an automorphisms $\theta$ of $\mathfrak{M}_{l}(\mathfrak{C})$ such that

$$
\left(\left[\left[\theta(\mu),[\mathfrak{x}, \mathfrak{y}]_{m}\right],[\mathfrak{x}, \mathfrak{y}]_{m}\right]\right)^{n}=\left[\left[\theta(\mu),[\mathfrak{x}, \mathfrak{y}]_{m}\right],[\mathfrak{x}, \mathfrak{y}]_{m}\right]
$$

is a generalized polynomial identity of $\mathfrak{R}$. This shows that $\theta(\mu)$ is a diagonal matrix for all $\theta \in \operatorname{Aut}\left(\mathfrak{M}_{l}(\mathfrak{C})\right)$. In particular, let $r \neq t$ and

$$
\theta(x)=\left(1+e_{r t}\right) x\left(1-e_{r t}\right)=x+e_{r t} x-x r_{r t}-e_{r t} x e_{r t} .
$$

Put $\theta(\mu)=\sum \theta(\mu)_{i j}^{\prime} e_{i j}$ with $\theta(\mu)_{i j}^{\prime} \in \mathfrak{C}$. Since $\theta(\mu)$ is diagonal, we have $\theta(\mu)_{l t}^{\prime}=0$. Hence $\mu_{t t}-\mu_{l l}=0$ and we conclude that $\mu$ is a central matrix, a contradiction.

Now, suppose that $\operatorname{dim}_{\mathfrak{C}}(\mathfrak{V})=\infty$. Then we have

$$
\left[\left[\lambda,[\mathfrak{x}, \mathfrak{y}]_{m}\right],[\mathfrak{x}, \mathfrak{y}]\right]^{n}=\left[\left[\lambda,[\mathfrak{x}, \mathfrak{y}]_{m}\right],[\mathfrak{x}, \mathfrak{y}]_{m}\right],
$$

for all $\mathfrak{x}, \mathfrak{y} \in \mathcal{Q}$. Moreover, again by Martindale's Theorem [10], it follows that $\mathfrak{R}=\mathfrak{R C}$ is a primitive ring with $\operatorname{soc}(\mathcal{Q})=\mathfrak{H} \neq 0$ and $e \mathfrak{H}$ e is a simple central algebra finite dimensional over $\mathfrak{C}$, for any minimal idempotent element $e \in \mathfrak{H}$. Further we may assume that $\mathfrak{H}$ is non-commutative, otherwise $\mathcal{Q}$ must be commutative. Of course, $\mathfrak{H}$ satisfies

$$
\left[\left[\lambda,[\mathfrak{x}, \mathfrak{y}]_{m}\right],[\mathfrak{x}, \mathfrak{y}]\right]^{n}=\left[\left[\lambda,[\mathfrak{x}, \mathfrak{y}]_{m}\right],[\mathfrak{x}, \mathfrak{y}]_{m}\right] .
$$

As $\mathfrak{H}$ is a simple ring, so either $\mathfrak{H}$ is generated by its idempotents or $\mathfrak{H}$ does not contain any non-trivial idempotent element. In this first case, suppose that $\mathfrak{H}$ contains two minimal orthogonal idempotent elements $e$ and $e^{\prime}$, by the hypothesis, for $[\mathfrak{x}, \mathfrak{y}]_{m}=\left[e \mathfrak{x}, e^{\prime}\right]_{m}=e \mathfrak{x} e^{\prime}$, we have

$$
e \mathfrak{x} e^{\prime}(\lambda) e \mathfrak{x} e^{\prime}=0 .
$$

Therefore, we get $e^{\prime} \lambda e \mathfrak{x} e^{\prime} \lambda e \mathfrak{x} e^{\prime} \lambda e=0$ and hence by the primeness of $\mathfrak{R}$, we find that $e^{\prime} \lambda e=0$, where $e$ and $e^{\prime}$ are orthogonal idempotents element of rank 1. As $e$ of rank 1 , we have $e \lambda(1-2)=0$ and $(1-e) \lambda e=0$, that is, $e \lambda=e \lambda e=\lambda e$. Therefore, $[\lambda, e]=0$ and $[\lambda, \mathfrak{H}]=0$, where $\mathfrak{H}$ is generated by these idempotent elements. This argument gives that $\lambda \in \mathfrak{C}$ or $\mathfrak{R}$ is commutative. In the other case, we conclude with contradiction. 
Thus, we take the case when $\mathfrak{H}$ cannot contain two minimal orthogonal idempotent elements and so, $\mathfrak{H}=\mathfrak{G}$ for suitable division ring $\mathfrak{G}$ finite dimensional over its center. This implies that $\mathfrak{G}=\mathfrak{H}$ and $\lambda \in D a$. By [12, Theorem 2.3.29] ([8, Lemma 2]), $\mathfrak{H} \subseteq \mathfrak{M}_{l}(\mathfrak{K}), \mathfrak{M}_{l}(\mathfrak{K})$ satisfies

$$
\left[\left[\lambda,[\mathfrak{x}, \mathfrak{y}]_{m}\right],[\mathfrak{x}, \mathfrak{y}]\right]^{n}=\left[\left[\lambda,[\mathfrak{x}, \mathfrak{y}]_{m}\right],[\mathfrak{x}, \mathfrak{y}]_{m}\right],
$$

where $\mathfrak{K}$ is a field. If $m=1$, then $\mathfrak{H} \subseteq \mathfrak{K}$, a contradiction. Moreover, if $m \geq 2$, then $\lambda \in Z\left(\mathfrak{M}_{l}(\mathfrak{K})\right)$, as we have just seen.

Finally, consider if $\mathfrak{H}$ does not contain any non-trivial idempotent element, then $\mathfrak{H}$ is finite dimensional division ring, that is, $\mathfrak{H}$ is commutative field and so $R$ is commutative too. If $\mathfrak{H}$ is infinite, then $\mathfrak{H} \otimes_{\mathfrak{C}} \mathfrak{K} \cong \mathfrak{M}_{l}(\mathfrak{K})$, where $\mathfrak{K}$ is a splitting field of $\mathfrak{H}$. The previous argument say that $\lambda \in Z(\mathfrak{R})$.

Assume that $\mathfrak{F}$ is not $\mathcal{Q}$-inner. Note that

$$
\begin{aligned}
\mathfrak{F}\left([\mathfrak{x}, \mathfrak{y}]_{m}\right)= & \sum_{k=1}^{m}(-1)^{k}\left(\begin{array}{c}
m \\
k
\end{array}\right)\left(\sum_{i+j=k-1} \mathfrak{y}^{i} \mathfrak{F}(\mathfrak{y}) \mathfrak{y}^{j}\right) \mathfrak{x}^{m-k} \\
& +\sum_{k=0}^{m}(-1)^{k}\left(\begin{array}{c}
m \\
k
\end{array}\right) \mathfrak{y}^{k} \mathfrak{F}(\mathfrak{x}) \mathfrak{y}^{m-k} \\
& +\sum_{k=0}^{m-1}(-1)^{m}\left(\begin{array}{c}
m \\
k
\end{array}\right) \mathfrak{y}^{k} \mathfrak{x}\left(\sum_{r+s=m-k-1} \mathfrak{y}^{r} \mathfrak{F}(\mathfrak{y}) \mathfrak{y}^{s}\right) .
\end{aligned}
$$

In view of our hypothesis and well-known results, we can say that $\mathfrak{R}$ satisfies

$$
\left[\mathfrak{F}\left([\mathfrak{x}, \mathfrak{y}]_{m}\right),[\mathfrak{x}, \mathfrak{y}]_{m}\right]^{n}=\left[\mathfrak{F}\left([\mathfrak{x}, \mathfrak{y}]_{m}\right),[\mathfrak{x}, \mathfrak{y}]_{m}\right],
$$

which is rewritten as

$$
\begin{aligned}
& \left(\left[\sum_{k=1}^{m}(-1)^{k}\left(\begin{array}{c}
m \\
k
\end{array}\right)\left(\sum_{i+j=k-1} \mathfrak{y}^{i} \mathfrak{F}(\mathfrak{y}) \mathfrak{y}^{j}\right) \mathfrak{x}^{m-k}+\sum_{k=0}^{m}(-1)^{k}\left(\begin{array}{c}
m \\
k
\end{array}\right) \mathfrak{y}^{k} \mathfrak{F}(\mathfrak{x}) \mathfrak{y}^{m-k}\right.\right. \\
& \left.\left.+\sum_{k=0}^{m-1}(-1)^{m}\left(\begin{array}{c}
m \\
k
\end{array}\right) \mathfrak{y}^{k} \mathfrak{x}\left(\sum_{r+s=m-k-1} \mathfrak{y}^{r} \mathfrak{F}(\mathfrak{y}) \mathfrak{y}^{s}\right), \sum_{k=0}^{m}(-1)^{k}\left(\begin{array}{c}
m \\
k
\end{array}\right) \mathfrak{y}^{k} \mathfrak{x y}^{m-k}\right]\right)^{n} \\
& =\left[\sum_{k=1}^{m}(-1)^{k}\left(\begin{array}{c}
m \\
k
\end{array}\right)\left(\sum_{i+j=k-1} \mathfrak{y}^{i} \mathfrak{F}(\mathfrak{y}) \mathfrak{y}^{j}\right) \mathfrak{x} \mathfrak{y}^{m-k}+\sum_{k=0}^{m}(-1)^{k}\left(\begin{array}{c}
m \\
k
\end{array}\right) \mathfrak{y}^{k} \mathfrak{F}(\mathfrak{x}) \mathfrak{y}^{m-k}\right. \\
& \left.+\sum_{k=0}^{m-1}(-1)^{m}\left(\begin{array}{c}
m \\
k
\end{array}\right) \mathfrak{y}^{k} \mathfrak{x}\left(\sum_{r+s=m-k-1} \mathfrak{y}^{r} \mathfrak{F}(\mathfrak{y}) \mathfrak{y}^{s}\right), \sum_{k=0}^{m}(-1)^{k}\left(\begin{array}{c}
m \\
k
\end{array}\right) \mathfrak{y}^{k} \mathfrak{x y}^{m-k}\right] .
\end{aligned}
$$


In the light of Kharchenko's Theorem [7], we find that

$$
\begin{aligned}
& \left(\left[\sum_{k=1}^{m}(-1)^{k}\left(\begin{array}{c}
m \\
k
\end{array}\right)\left(\sum_{i+j=k-1} \mathfrak{y}^{i} \mathfrak{w} \mathfrak{y}^{j}\right) \mathfrak{x y}^{m-k}+\sum_{k=0}^{m}(-1)^{k}\left(\begin{array}{c}
m \\
k
\end{array}\right) \mathfrak{y}^{k} \mathfrak{z}^{m-k}\right.\right. \\
+ & \left.\left.\sum_{k=0}^{m-1}(-1)^{m}\left(\begin{array}{c}
m \\
k
\end{array}\right) \mathfrak{y}^{k} \mathfrak{x}\left(\sum_{r+s=m-k-1} \mathfrak{y}^{r} \mathfrak{w} \mathfrak{y}^{s}\right), \sum_{k=0}^{m}(-1)^{k}\left(\begin{array}{c}
m \\
k
\end{array}\right) \mathfrak{y}^{k} \mathfrak{x} \mathfrak{y}^{m-k}\right]\right)^{n} \\
= & {\left[\sum_{k=1}^{m}(-1)^{k}\left(\begin{array}{c}
m \\
k
\end{array}\right)\left(\sum_{i+j=k-1} \mathfrak{y}^{i} \mathfrak{w} \mathfrak{y}^{j}\right) \mathfrak{x y}^{m-k}+\sum_{k=0}^{m}(-1)^{k}\left(\begin{array}{c}
m \\
k
\end{array}\right) \mathfrak{y}^{k} \mathfrak{z} \mathfrak{y}^{m-k}\right.} \\
+ & \left.\sum_{k=0}^{m-1}(-1)^{m}\left(\begin{array}{c}
m \\
k
\end{array}\right) \mathfrak{y}^{k} \mathfrak{x}\left(\sum_{r+s=m-k-1} \mathfrak{y}^{r} \mathfrak{w} \mathfrak{y}^{s}\right), \sum_{k=0}^{m}(-1)^{k}\left(\begin{array}{c}
m \\
k
\end{array}\right) \mathfrak{y}^{k} \mathfrak{x} \mathfrak{y}^{m-k}\right]
\end{aligned}
$$

for all $\mathfrak{x}, \mathfrak{y}, \mathfrak{w}, \mathfrak{z} \in \mathfrak{R}$, and hence, it satisfied by $\mathcal{Q}$ [1, Theorem 6.4.4]. Thus, $\mathcal{Q}$ is non-commutative as $\mathfrak{R}$. Let us take $\delta \in \mathcal{Q}$ with $\delta \notin \mathfrak{C}$. Also, we can see that $\phi: \mathcal{Q} \rightarrow \mathcal{Q}$ is a nonzero derivation of $\mathcal{Q}$ defined by $\phi(\mathfrak{x})=[\delta, \mathfrak{x}]$ for all $\mathfrak{x} \in \mathcal{Q}$. Replacing $\mathfrak{w}, \mathfrak{z}$ by $\phi(\mathfrak{x}), \phi(\mathfrak{y})$ in the last expression, we obtain that

$$
\left[\phi\left([\mathfrak{x}, \mathfrak{y}]_{m}\right),[\mathfrak{x}, \mathfrak{y}]_{m}\right]^{n}=\left[\phi\left([\mathfrak{x}, \mathfrak{y}]_{m}\right),[\mathfrak{x}, \mathfrak{y}]_{m}\right]
$$

for all $\mathfrak{x}, \mathfrak{y} \in \mathcal{Q}$. This can be rewritten as

$$
\left[\left[\delta,[\mathfrak{x}, \mathfrak{y}]_{m},[\mathfrak{x}, \mathfrak{y}]_{m}\right]^{n}=\left[\left[\delta,[\mathfrak{x}, \mathfrak{y}]_{m},[\mathfrak{x}, \mathfrak{y}]_{m}\right] .\right.\right.
$$

The last expression is the same as (1).

Using same technique, we get the required conclusion.

We immediately get the following corollary as the consequence of above theorem.

Corollary 1. Let $\mathfrak{R}$ be a prime ring of characteristic different from two and $\mathfrak{F}$ be a derivation of $\mathfrak{R}$ such that

$$
\left[\mathfrak{F}\left([\mathfrak{x}, \mathfrak{y}]_{m}\right),[\mathfrak{x}, \mathfrak{y}]_{m}\right]^{n}=\left[\mathfrak{F}\left([\mathfrak{x}, \mathfrak{y}]_{m}\right),[\mathfrak{x}, \mathfrak{y}]_{m}\right]
$$

for all $\mathfrak{x}, \mathfrak{y} \in \mathfrak{R}$, where $m, n$ are positive integers. Then $\mathfrak{R}$ is commutative.

\section{REFERENCES}

[1] K. I. Beidar, W. S. Martindale III, A. V. Mikhalev, Rings with Generalized Identities, Pure and Applied Mathematics, Marcel Dekker 196, New York, 1996.

[2] C. L. Chuang, GPIs having coefficients in Utumi quotient rings, Proceedings of the American Mathematical Society, 103 (1988), 723-728.

[3] A. Giambruno, J. Z. Goncalves, A. Mandel, Rings with algebric n-Engel elements, Communications in Algebra, 22 (5) (1994), 1685-1701.

[4] I. N. Herstein, A condition for the commuttivity of the rings, Canadian Journal of Mathematics, 9 (1957), 583-586. 
[5] S. Huang, Derivation with engel conditions in prime and semiprime rings, Czechoslovak Mathematical Journal, 61 (136) (2011), 1135-1140.

[6] N. Jacobson, Structure theory for algebraic algebras of bounded degree, Annals of Mathematics, 46 (4) (1945), 695-707.

[7] V. K. Kharchenko, Differential identities of prime rings, Algebra Logic, 17 (1979), 155-168.

[8] C. Lanski, An Engel condition with derivation, Proceedings of the American Mathematical Society, 118 (1993), 731-734.

[9] T. K. Lee, Semiprime rings with differential identities, Bulletin of the Institute of Mathematics Academia Sinica, 20 (1992), 27-38.

[10] W. S. Martindale III, Prime rings satisfying a generalized polynomial identity, Journal of Algebra, 12 (1969), 576-584.

[11] M. A. Raza, N. Rehman, A note on an Engel condition with derivations in rings, Creative Mathematics and Informatics, 26 (1) (2017), 19-27.

[12] L. Rowen, Polynomial identities in ring theory, Pure and Applied Mathematics, Vol. 84, Academic Press, New York, 1980.

\section{NADEEM UR REHMAN}

Department of Mathematics

Aligarh Muslim University

AligarH-202002

INDIA

E-mail address: nu.rehman.mm@amu.ac.in 